\title{
THE U.S. RARE ISOTOPE ACCELERATOR PROJECT
}

\author{
G. Savard, Physics Division, Argonne National Laboratory
}

\begin{abstract}
The Rare Isotope Accelerator (RIA) is a next generation radioactive beam facility in preparation in the US. RIA combines the best of standard ISOL and in-flight fragmentation technology with novel approaches to handle high-primary beam power and remove existing limitations in the extraction of short-lived isotopes. The use of a versatile primary accelerator (superconducting linac designed to allow multiple charge state acceleration and capable of providing beams from protons at $900 \mathrm{MeV}$ to uranium at $400 \mathrm{MeV} / \mathrm{u}$ at power levels up to $400 \mathrm{~kW}$ ) allows various production and extraction schemes to be used to optimize production of specific isotopes. In particular, a novel approach where radioactive isotopes produced by fragmentation of a fast heavy-ion beam are stopped and cooled in a high-purity helium gas cell and extracted by electromagnetic fields as thermal singly charged ions promises huge increases in efficiency for very short-lived isotopes. These isotopes will then be available for studies at ion source energy or can be further accelerated by a second superconducting linac whose novel injection system, based on $\mathrm{CW}$ low-Frequency RFQs, allows the efficient acceleration of singly-charged heavy ions with masses up to 240 amu from ion source energy. The high-intensity radioactive beams at RIA will be made available to four experimental areas spanning the energy regime from ion source energy to primary beam energy.
\end{abstract}

\section{INTRODUCTION}

The study of the properties and reactions of short-lived isotopes is a key to our understanding of fundamental questions in nuclear physics, nuclear astrophysics and the study of fundamental interactions at low-energy. This has long been recognized by the international nuclear physics community and is reflected by the investments in that field that have been or are being made in North America, Europe and Asia. The US nuclear physics community recognizes the importance of such studies and stated in its 1996 long range plan that a next generation ISOL type facility is its highest priority item for new construction after the completion of RHIC. The recent NSAC 2001 long range plan reiterates this conclusion and supports the construction of a facility with an enlarged scope, the Rare Isotope Accelerator (RIA) facility, as the highest priority for new construction in nuclear physics. The RIA facility incorporates new technologies that make it significantly more powerful than any existing or planned facilities in that field. These enhanced capabilities come from a combination of factors:
- Use of a variety of production techniques for short-lived isotopes including new approaches that remove the main previous limitations of standard ISOL techniques

- Powerful superconducting driver linac capable of accelerating any stable ion from proton to uranium and designed to allow simultaneous acceleration of multiple charge states of a given ion to attain unprecedented power for the heaviest ions

- Very efficient post-acceleration scheme based on a superconducting linac injected by low frequency RFQs capable of accelerating charge +1 radioactive ions of mass up to 240 amu from ion source energy

- First rate experimental equipment and multiple user capability to maximize use of the facility.

The concept for this facility and the status of the development will be briefly described below. A possible timescale and expected cost for the facility completion will also be presented.

\section{FACILITY DESCRIPTION}

A simplified schematic layout of the RIA facility is shown in figure 1 . The main functional components are described in the following.

\subsection{Driver Accelerator}

The driver linac produces the primary high-power stable ion beam required to produce the radioisotopes. The RIA facility will use a number of production mechanisms spanning the range from spallation on thick targets which requires high energy light ion beams ( $\mathrm{p}, \mathrm{d}$, ${ }^{3} \mathrm{He}$ at energies close to a $\mathrm{GeV}$ ), fragmentation which requires heavy-ion beams at high-intensity and energies in the $100-1000 \mathrm{MeV} / \mathrm{u}$ range for mid-mass nuclei, to inflight fission which requires high intensity uranium beams at energies up to about $400 \mathrm{MeV} / \mathrm{u}$. To be able to fully exploit the various production mechanisms available at RIA it is therefore imperative that the driver linac be capable of accelerating essentially all stable nuclei to energies above $400 \mathrm{MeV} / \mathrm{u}$ with high efficiency. The broad mass-to-charge ratio of the species to be accelerated together with the higher energy for lighter ions and high current requirements dictate the use of a superconducting linac whose short independently-phased cavities allow for efficient acceleration over a wide velocity profile.

The primary beam intensity requirements specify a beam power of $100 \mathrm{~kW}$ or higher for all primary species. While this is achievable with standard techniques for light ions, for the heaviest ions such as uranium, the present 
performance of even the best existing ECR sources would limit conventional accelerators to power well below that. This limitation is overcome in the RIA driver by accelerating multiple charge states [1] of the heavy ions of interest through the linac making use of the large acceptance of the superconducting linac. Taking as an example the most difficult beam, uranium at $400 \mathrm{MeV} / \mathrm{u}$, we would find two charge states of uranium from an advanced ECR source [2], 28+ and 29+, transported from the ion source platform, bunched at $28.7 \mathrm{MHz}$ and the 2 charge states loaded into successives buckets of the 57.5 MHz RFQ. After the RFQ, both charge states are injected in the low-beta section of the superconducting linac where they are accelerated up to $9.3 \mathrm{MeV} / \mathrm{u}$ where the ions are stripped to a mean charge state of about +72 and 5 charge states (from +69 to +73 ) are injected into and accelerated by the medium-beta section of the linac. At an energy of roughly $83 \mathrm{MeV} / \mathrm{u}$ the ions are further stripped and 3 charge states (charge state +87 to +89 ) are further accelerated in the high-beta section of the linac and delivered to the production stations. The 5 charge states and 3 charge states after the first and second strippers contain most of the intensity of the beam and the full process is then performed with a reasonable size linac (total accelerating voltage of $1.4 \mathrm{GV}$ ) and without the usual losses associated with stripping stages. The different types of resonators used in the driver linac are shown in figure 2. The beam characteristics range from proton at $900 \mathrm{MeV}$ and power up to $400 \mathrm{~kW}$ (limited by RF power) to uranium at $400 \mathrm{MeV} / \mathrm{u}$ and power up to about $170 \mathrm{~kW}$, limited by present ECR ion source performance.

\section{Driver Linac ( to $400 \mathrm{MeV} /$ nucleon)}

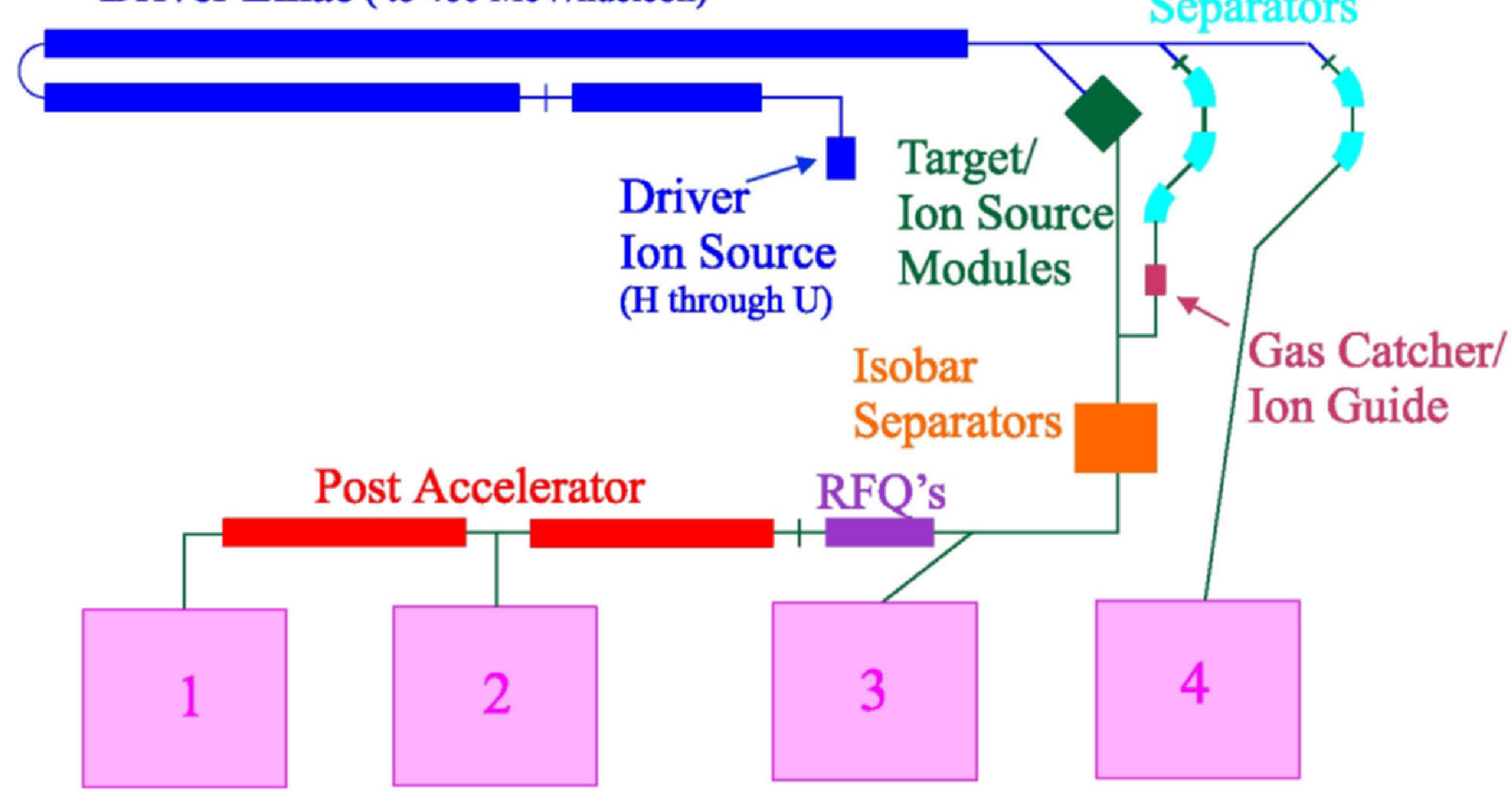

\section{Experimental Areas:}

\section{$1:<12 \mathrm{MeV} / \mathrm{u} \quad$ 2: $<1.5 \mathrm{MeV} / \mathrm{u} . \quad 3:$ Nonaccelerated}

\section{4: In-flight fragments}

Figure 1: Simplified schematic layout of the Rare Isotope Accelerator (RIA) facility.

The design of the driver accelerator took a number of bold steps to meet the requirements of the RIA facility. A vigorous $R \& D$ program led to the development of the multiple charge state acceleration concept [1], its experimental demonstration at the ATLAS facility at ANL [3], the development of the spoke-cavity superconducting structures [4] to fill the gap in the velocity regime between the low- $\beta$ ATLAS type cavities and the CEBAF type velocity-of-light structures, development of the SNS high- $\beta$ cavities [5] that will be used for the higher energy section of the linac and finally the development of windowless thin film liquid lithium stripper foils [6] that is ongoing at ANL
An RF switchyard will be located at the end of the linac to allow pulse-to-pulse sharing of the primary beam between target stations. This is actually non-trivial because of the large emittance of the heavy beams after multiple charge state acceleration but it is found that an initial separation stage made of a normal conducting cavity can yield sufficient spatial separation. The very high beam power available will permit two or more targets to received the full $100 \mathrm{~kW}$ beam for which they are specified simultaneously. This will enhance the multiple user capabilities of this facility that are required to service the large user community. 


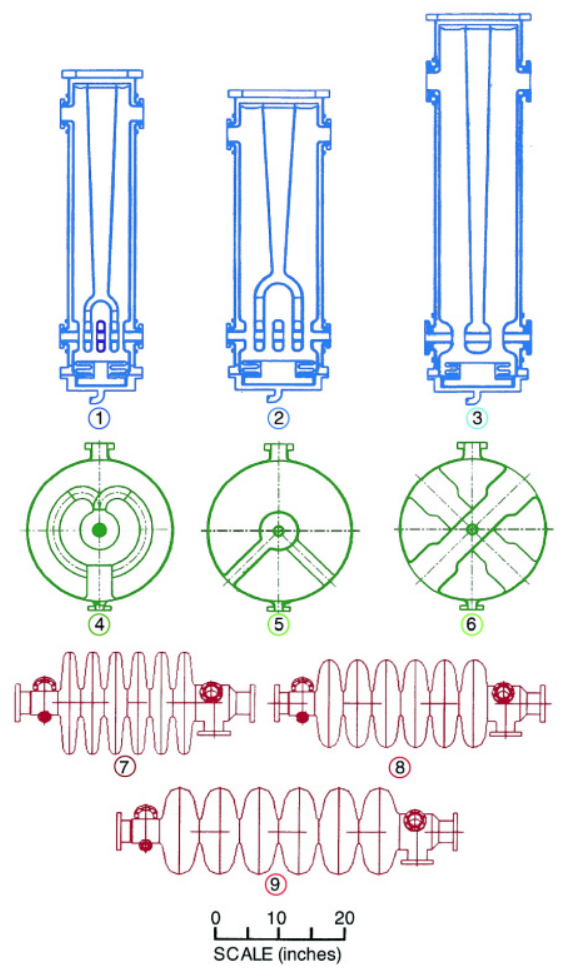

Fig. 2 Views of the nine types of superconducting resonators used in the RIA driver linac.

\subsection{Isotope Production complex}

The production of the radioisotopes will occur by interaction of the primary beams on high-power targets located in an enclosed production building. Remote handling capabilities and the required radiation control mechanisms will be present in this building. The radioactive isotope inventory that will be present in this building require it to be a category 3 non-reactor nuclear facility and all required conditions for operation of such a facility will be met. The production of radioactive isotopes in this complex can occur through 4 main mechanisms, all of which are enabled by the versatile driver available at RIA, which are described below.

The standard ISOL technique where a high energy light ion beam impinges on a thick target producing short-lived isotopes via spallation or induced-fission reactions. The target is heated to release the activity quickly. The activity then diffuses to an ion source where it is ionized and extracted and made available for experiments at low energy or at higher energy after post acceleration. A second approach is that of production of radioactive species by fragmentation of a fast heavy ion beam on a thin target capable of handling the high beam power. The reaction products are pushed forward by the kinematics and can be separated from the beam in a fragment separator. These beams are then available for experiments at high-energy but with rather poor beam quality.

These two conventional approaches are implemented with unprecedented power at RIA where new technology based on flowing liquid lithium targets [7] will allow the full driver beam power to be used effectively. These two approaches are supplemented by two new complimentary approaches that remove the main limitations of the standard approaches. The first new approach is the twostep neutron generator technique [7] where the highenergy light ion beam is converted to fast neutrons in a cooled converter which is surrounded by a production target where fissions induced by the fast neutrons produce the radioactive species which are then extracted in a fashion similar to that used in the standard ISOL technique. This yields significant gains since the power deposited by the light ion beam (mostly via electromagnetic interactions) is in a volume which can be cooled efficiently, while only the neutron and the fission power is deposited in the releasing target where power removal is much more difficult to perform without affecting the other functions of the device.

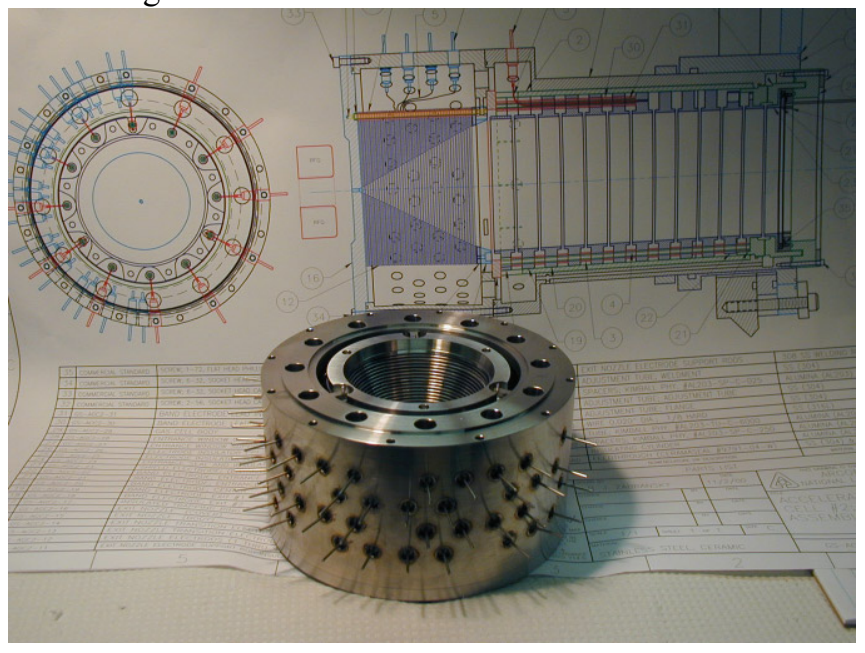

Fig. 3 Extraction region of the quarter scale gas cell RIA prototype successfully tested at ANL. In the background is a drawing of the full gas cell system. This system reached an efficiency of $45 \%$ with delay times as short as 7 milliseconds.

A second new approach brings together the advantages of the in-flight fragmentation approach with that of the ISOL techniques. Radioactive isotopes are produced by fragmentation (or in-flight fission) of fast heavy-ion beams on a "thick" thin target, the reaction products are pushed again forward by the kinematics and after selection by a large acceptance fragment separator are slowed down and stopped in a gas catcher system [8] where they are thermalized but remain singly charged and can be extracted by a combination of DC and RF fields to be further reaccelerated. This results in beams of quality similar to those obtained by ISOL techniques, available at any energy, without the chemical limitations encountered by the ISOL technique in the diffusion and release out of thick targets.

Again, a vigorous $R \& D$ program is ongoing on these different topics with yield calculations [9] for the different mechanisms, high-power ISOL type target development 
[10], thermal and flow calculations for the high-power fragmentation targets which will use liquid lithium technology [7] and for which a prototype system is under construction. The new approach of stopping the fast beams in a large high-purity gas cell system and fast extraction by RF \& DC fields is also being thoroughly investigated with a demonstration with a quarter scale system (see figure 3) that exceeded the initial RIA efficiency requirements by more than a factor of two [11] and a full scale prototype now under construction that will be fully characterized at ANL before a test at the full RIA energy [12] that will be conducted at GSI.

\subsection{Post-Accelerator}

The most efficient production mechanisms for slow radioactive ions yield these ions in the $1+$ charge state. The post-accelerator must therefore be able to accept such low charge-to-mass ratio ions from ion source energy. One approach to this problem is to increase the charge state of the ions before acceleration via a charge booster stage, be it an ECR type device or an EBIT based system. While both of these approaches have shown significant progress over the last decade, for a versatile system they present too low an efficiency at this point. To ensure maximum efficiency in the post-acceleration process, RIA proposes a different approach [13] based on a superconducting linac accelerator injected by low frequency RFQs.

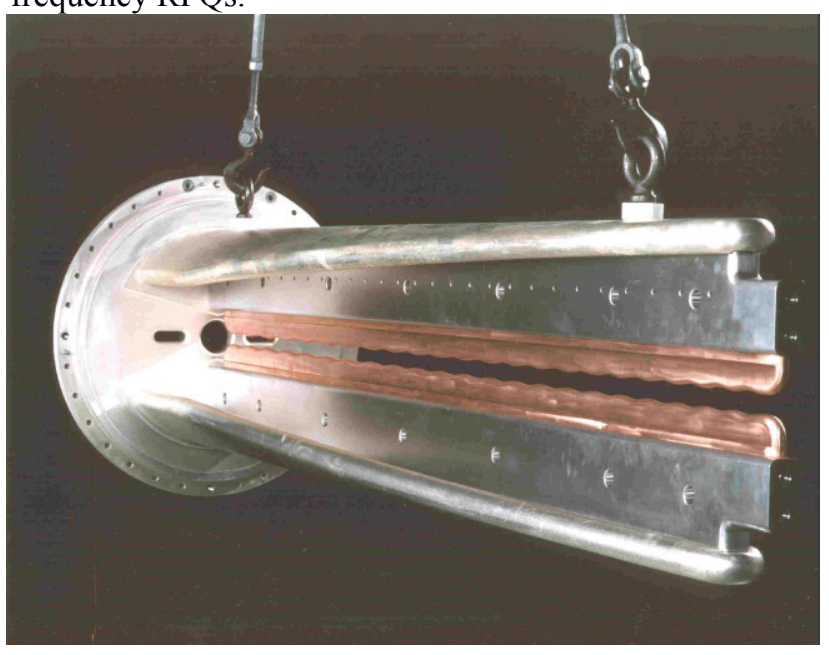

Fig. 4 View of 2 vanes of the $12 \mathrm{MHz} \mathrm{CW}$ split-coax RFQ developed for the acceleration of very low chargeto-mass radioactive ion beams from ion source energy.

The singly charged radioactive ions extracted from the ion source are first mass separated by a high-resolution $(\Delta \mathrm{m} / \mathrm{m}=1 / 20000)$ isobar separator [14]. The selected isotopes are then bunched by a multi-harmonic buncher and accelerated through a series of 3 low frequency RFQs, the first two located on a variable voltage highvoltage platform. The first RFQ is a CW split-coax RFQ structure (figure 4) [15] recently developed at Argonne and capable of accelerating ions with mass-to-charge ratio as high as 240. The second and third RFQ are of a novel hybrid structure [16] mixing RFQ and IH type structures to obtain higher accelerating gradient with still enough focusing force. The following low beta superconducting linac section can accept ions of $\beta=0.011$ with $\mathrm{q} / \mathrm{m} \geq 1 / 66$ so that ions with mass larger than 66 must be stripped. This is done with non-equilibrium charge state stripping [17] in helium gas at two possible location: ions of mass in the range $66<\mathrm{A}<132$ are stripped to charge state +2 with close to $50 \%$ efficiency on the high-voltage platform between the first two RFQs, ions with mass above 132 are stripped to charge state +3 or +4 before the third RFQ section with efficiency in the $30 \%$ range.

The ions are then accelerated at that charge state by low beta interdigital superconducting cavities up to an energy of $600 \mathrm{keV} / \mathrm{u}$ after which they are further stripped to $\mathrm{q} / \mathrm{m}$ $\geq 0.12$ for further acceleration in ATLAS type superconducting cavities. The beam is therefore available at all energies from ion source energy up to energies above $10 \mathrm{MeV} / \mathrm{u}$ for mid-mass ions. The acceptance of all parts of the linac being very large compared to typical emittance from ISOL sources, the transmission is also very high, limited by the bunching efficiency (about 85\%) and the stripping for the heavier beams.

\subsection{Experimental Areas}

RIA will host four experimental areas corresponding to different energy regimes for the radioactive ion beams. The ions produced by the three mechanisms leading to low energy radioactive ions will be available directly at ion source energy to a low energy experimental area for decay spectroscopy, ion trap work, laser spectroscopy or implantation at low energy. These ions will also be available for acceleration to an astrophysics experimental area where beam with energies up to about $1.5 \mathrm{MeV} / \mathrm{u}$ will be available for reaction experiments at regime relevant to quiescent and explosive astrophysical processes. They will also be available at Coulomb barrier energy in a nuclear structure experimental area where beams with energy up to about $12 \mathrm{MeV} / \mathrm{u}$ will be available.

In addition, radioactive beams produced by fragmentation and separation at high energy will be available directly for experiment at energies of about 400 $\mathrm{MeV} / \mathrm{u}$. This area will concentrate on short-lived isotopes with very low yield using the fact that the ability at high energy to use thicker targets enhances the sensitivity for certain types of experiments.

The research to be pursued in these experimental areas requires sophisticated new instruments. The instruments necessary for the physics to be performed in the first three experimental areas were discussed in a workshop organized by LBNL, ORNL and ANL in the summer of 1998 [18]. The experimental equipment required in the high-energy experimental area is described in a recent white paper on the physics opportunities with fast beams at RIA [19]. Possible applications of the beams made 
available by RIA were also studied [20] and can be accommodated in these experimental areas.

\section{EXPECTED PERFORMANCE}

The proposed RIA facility will provide yields of shortlived isotopes far in excess of those available at existing facilities. This is due to the high primary beam power intensity, the flexibility in the primary beam selection which allows the best production mechanism to be used, the new target technologies that allow the normal limitations of ISOL type systems to be overcome, and finally the use of the most efficient post-acceleration scheme proposed to date.

The maximum yield available for post-acceleration will exceed $10^{12}$ radioactive ions per second for isotopes best produced by standard ISOL techniques and most importantly will show important yield (up to $10^{9}$ per second) even for isotopes of elements not reachable by standard approaches. These yields have been calculated for the different reaction mechanisms and are available on the web at http://www.phy.anl.gov/ria.

Similarly, the fast beam facility will benefit from the highest intensity heavy-ion beam driver, the high-power liquid lithium target technology and a high-acceptance fragment separator to obtain the highest radioactive beam yields in the high-energy experimental area. The fast beam yields are available on the same web site.

\section{COST AND SCHEDULE}

A site independent costing of the facility was undertaken jointly by ANL and NSCL and reviewed by an NSAC subcommittee. It was found that the cost of the facility, including the required experimental equipment, a fragmentation facility and a $30 \%$ contingency, was $\$ 644 \mathrm{M}$ in fixed FY01 dollars. Adding the cost of the R\&D, the CDR and environmental studies, and finally the preoperation, yields a total project cost of $\$ 834 \mathrm{M}$. The facility would operate with close to 400 full-time staff at an operating cost of about $\$ 75 \mathrm{M}$ per year.

The timescale for construction is set by final approval of the facility, site selection, CDR and environmental studies. A start of construction in FY2004 is expected in which case the facility would become operational in FY2009. A national program for RIA R\&D with current participation from ANL, JLAB, LANL, LBNL, LLNL, NSCL and ORNL is further developing and performing value engineering on key components of the facility.

\section{ACKNOWLEDGEMENTS}

The RIA concept has evolved from ideas generated within the US nuclear physics community and contributions from abroad and from other fields. This work was supported by the US Department of Energy, Division of Nuclear Physics, under contract number W31-109-ENG-38.

\section{REFERENCES}

[1] P.N. Ostroumov and K.W. Shepard, Phys. Rev. ST Accel. Beams 3 (2000) 030101.

[2] C.M. Lyneis et al, Proc. 2001 International conference on cyclotron and their applications, East Lansing, Michigan, May 12-17 2001.

[3] P.N. Ostroumov et al, "Simultaneous acceleration of multiply charged ions through a superconducting linac", PRL 86 (2001) 2798.

[4] K. Shepard et al, "Superconducting intermediatevelocity drift-tube cavities for the RIA driver linac", These proceedings.

[5] P. Kneisel et al, "Superconducting prototype cavities for the Spallation Neutron Source (SNS) project", These proceedings.

[6] J.A. Nolen et al, "Studies of liquid lithium films for high-intensity ion beam strippers", Argonne Physics Division 2000 Annual Report.

[7] J.A. Nolen et al, "Liquid-lithium cooling for $100 \mathrm{~kW}$ ISOL and fragmentation targets", proceedings of the RNB5 conference, Divonne, France, April 2000.

[8] G. Savard et al, "ISOL beams from fragmentation: the best of both worlds", proceedings of the RNB5 conference, Divonne, France, April 2000.

[9] http://www.phy.anl.gov/ria/

[10] G. Alton et al, "Design and characterization of high power targets for RIB generation", These proceedings.

[11] G. Savard et al, "Development of a large accelerated gas cell system for the collection of fast recoiling radioactive ions", report ANL-00/20, Argonne Physics Division 1999 Annual Report, p. 104-107.

[12] G. Savard et al, "Experiments on the conceptual design of a gas-catcher system for in-flight-separated exotic nuclear beams as a component of a nextgeneration facility", Proposal S258 to the Dec. 2000 GSI program advisory committee.

[13] P.N. Ostroumov et al, "Design of a post accelerator for the rare isotope accelerator facility", These proceedings.

[14] M. Portillo et al, "Design layout of an isobar separator based on $5^{\text {th }}$ order calculations", These proceedings.

[15] M. Kelly et al, "Singly-charged heavy-ion beam studies on a $12 \mathrm{MHz}$ RFQ", These proceedings.

[16] P.N. Ostroumov and A.A. Kolomiets, "New concept for acceleration of slow low-charge-state heavy ion beams", These proceedings.

[17] P. Decrock et al, "Low energy stripping of $\mathrm{Kr}+, \mathrm{Xe}+$ and $\mathrm{Pb}+$ beams in helium and nitrogen", Rev. Sci. Instr. 68 (1997) 2322.

[18] "Experimental equipment for an advanced ISOL facility". Workshop held at LBNL, Berkeley, Ca, July 22-25, 1998.

[19] http://www.nscl.msu.edu/research/ria/whitepaper.pdf [20] "RIA applications workshop", Los Alamos, New Mexico, October 30-31 2000, report LA-UR 01-1008. 